\title{
Erektile Dysfunktion bei Männern in der zweiten Lebenshälfte
}

\author{
Buddeberg, C ; Bucher, T ; Hornung, R
}

\begin{abstract}
Hintergrund: Bisherige Studien zur Prävalenz der erektilen Dysfunktion (ED) zeigen unterschiedliche Ergebnisse. Mit der vorliegenden Studie werden die Häufigkeit und graduelle Ausprägung der ED, deren begünstigende Faktoren sowie das Hilfesuchverhalten der Betroffenen untersucht. Stichprobe und Methodik: Eine Zufallsstichprobe von 628 Deutschschweizer Männern (Durchschnittsalter 61,5Jahre) wurde mittels Fragebogen zur sexuellen Funktionsfähigkeit, Komorbidität und zu ihrem Hilfesuchverhalten befragt. Ergebnisse: 9,6\% der Befragten berichten über eine vollständige, 18,0\% über eine moderate und 41,4\% über eine minimale ED. Alle Grade der Störung nehmen mit dem Alter zu und korrelieren mit somatischer bzw. psychischer Komorbidität sowie Medikamenteneinnahme. Nur 3,2\% geben an, schon Mittel zur Verbesserung der Erektionsfähigkeit eingenommen bzw. verwendet zu haben. Schlussfolgerungen: Nur ein kleiner Teil von Männern mit ED sucht ärztliche Hilfe. Sachliche Aufklärung und kompetente ärztliche Beratung von Betroffenen erscheinen angesichts der guten Wirksamkeit von PDE-5-Hemmern angebracht
\end{abstract}

DOI: https://doi.org/10.1007/s00120-005-0841-5

Posted at the Zurich Open Repository and Archive, University of Zurich ZORA URL: https://doi.org/10.5167/uzh-156269

Journal Article

Published Version

Originally published at:

Buddeberg, C; Bucher, T; Hornung, R (2005). Erektile Dysfunktion bei Männern in der zweiten Lebenshälfte. Der Urologe. Ausg. A, 44(9):1045-1051.

DOI: https://doi.org/10.1007/s00120-005-0841-5 
Urologe [A] 2005 - 44:1045-1051 DOI 10.1007/s00120-005-0841-5

Online publiziert: 10. Juni 2005

๑) Springer Medizin Verlag 2005

\section{Redaktion}

R. Hautmann, Ulm
C. Buddeberg ${ }^{1} \cdot$ T. Bucher ${ }^{2} \cdot$ R. Hornung ${ }^{2}$

${ }^{1}$ Abteilung Psychosoziale Medizin, Universitätsspital, Zürich, Schweiz $\cdot{ }^{2}$ Sozial- und Gesundheitspsychologie, Psychologisches Institut, Universität, Zürich, Schweiz

\section{Erektile Dysfunktion bei Männern in der zweiten Lebenshälfte}

\section{Hintergrund und Fragestellung}

Schon Masters und Johnson konnten in ihren grundlegenden sexualphysiologischen Untersuchungen zeigen, dass beim älter werdenden Mann die Erektionsfähigkeit abnimmt, die Erektion von geringerer Rigidität und kürzerer Dauer ist und die Refraktärzeit nach einem Orgasmus länger wird $[1,2]$. Neuere multinationale Studien zur Häufigkeit der erektilen Dysfunktion (ED) in der Durchschnittsbevölkerung zeigen unterschiedliche Prävalenzraten $[3,4,5,6]$.

Die z. T. beträchtlichen Differenzen in den Ergebnissen sind auf Unterschiede in der Definition einer ED, auf unterschiedliche Messinstrumente, unterschiedliche Altersverteilungen der Stichproben sowie Komorbiditäten der Befragten zurückzuführen [3, 4]. In der Mehrzahl der Studien werden durchschnittliche Prävalenzraten von $5-20 \%$ für mittelgradige bis schwere Formen von ED berichtet, wobei die Häufigkeit jenseits des 40. Altersjahres kontinuierlich zunimmt und bei $>60$-Jährigen zwischen $20-40 \%$ liegt [4].

Wie einzelne Studien zeigen, korreliert die Häufigkeit und das Ausmaß einer ED mit folgenden Determinanten: niedrigem sozioökonomischem Status, Rauchen, Komorbidität mit Erkrankungen des Urogenitaltraktes, Diabetes mellitus, des Nervensystems sowie des kardiovaskulären Systems und psychischen Störungen wie Depression, Panikstörung und Alkoholmissbrauch $[5,6,7,8,9,10,11,12,13]$.

Männer mit ED berichten über eine Beeinträchtigung ihrer Lebensqualität [14] sowie ihrer sexuellen Zufriedenheit und Aktivität [15]. Die subjektive Bewertung einer ED ist altersabhängig. Ältere Männer fühlen sich durch eine Einschränkung ihrer Erektionsfähigkeit weniger beeinträchtigt als jüngere [16]. Ergebnisse neuerer Studien deuten darauf hin, dass ca. 60\% der Männer mit einer ED unterschiedlichen Grades schon mit einem Arzt über ihre sexuelle Störung gesprochen haben, jedoch $<16 \%$ eine medikamentöse Therapie mit einem PDE-5-Hemmer anwenden $[4,5]$. Der Schweregrad der ED, subjektive Vorstellungen der Betroffenen über eine medikamentöse ED-Therapie sowie Einstellungen der Partnerinnen und der behandelnden Ärzte beeinflussen die (regelmäßige) Einnahme eines PDE-5-Hemmers $[17,18]$. Nicht selten sind es nach wie vor Schamgefühle, welche Betroffene daran hindern, therapeutische Hilfe $\mathrm{zu}$ suchen [19].

Auf 2 Studien zur Prävalenz der ED sei besonders hingewiesen, da die Ergebnisse der vorliegenden Studie mit diesen am ehesten zu vergleichen sind. In der Massachusetts Male Aging Study (MMAS) wurden Männer im Alter zwischen 40-70 Jahren befragt. $52 \%$ der Befragten berichteten über eine ED, wobei das Ausmaß der Störung (gering, mittelgradig, vollständig) mit dem Alter kontinuierlich zunahm [20].

In der Kölner Studie zur Männergesundheit (Cologne Male Survey CMS) wurden Männer im Alter zwischen 30-80 Jahren befragt. Als Kriterium für das Vorliegen einer ED wurde ein Summenscore verwendet. 19,2\% der Befragten berichteten über eine ED, deren Häufigkeit mit dem Alter ebenfalls zunahm [21]. In beiden Studien wurden Zusammenhänge der ED mit somatischen und psychischen Begleiterkrankungen berücksichtigt.

Hauptfragestellungen der vorliegenden Studie waren 1. die Häufigkeit einer ED in Abhängigkeit von Alter und Gesundheitszustand, 2. Zusammenhänge zwischen sexueller Funktionsfähigkeit, Aktivität und Zufriedenheit und 3. das Hilfesuchverhalten der Männer mit einer ED. Die Untersuchung wurde im Rahmen eines größeren Forschungsprojekts durchgeführt, in welchem >45-jährige Männer und Frauen zu verschiedenen Aspekten ihres Sexuallebens befragt wurden $[22,23]$.

\section{Studiendesign und Methodik}

Im Rahmen einer Studie zur „Gesundheit, Lebenszufriedenheit und Sexualität bei Menschen ab 45 Jahren“ wurde im Herbst 1999 eine Zufallsstichprobe von Personen der deutschsprachigen Schweiz mittels eines per Post zugeschickten Fragebogens u. a. auch zu ihrem Sexualleben befragt [23]. Im Fragebogen für die männlichen Studienteilnehmer wurden zur Erfassung der sexuellen Funktionsfähigkeit dieselben Fragen verwendet wie in der MMAS. Einzig der Begriff „Impotenz“, der in der MMAS z. T. verwendet wurde, ist in der vorliegenden Studie durch „Erektionsfähigkeit“ ersetzt. Der Wortlaut der Frage war: „Mit zunehmendem Alter kann auch

Gefördert durch Schweizerischer Nationalfonds NF-Nr. 32-52628.97. 
Urologe [A] 2005 * 44:1045-1051

DOI 10.1007/s00120-005-0841-5

(C) Springer Medizin Verlag 2005

\section{Buddeberg $\cdot$ T. Bucher $\cdot$ R. Hornung}

\section{Erektile Dysfunktion bei Männern in der zweiten Lebenshälfte}

\section{Zusammenfassung}

Hintergrund. Bisherige Studien zur Prävalenz der erektilen Dysfunktion (ED) zeigen unterschiedliche Ergebnisse. Mit der vorliegenden Studie werden die Häufigkeit und graduelle Ausprägung der ED, deren begünstigende Faktoren sowie das Hilfesuchverhalten der Betroffenen untersucht.

Stichprobe und Methodik. Eine Zufallsstichprobe von 628 Deutschschweizer Männern (Durchschnittsalter 61,5 Jahre) wurde mittels Fragebogen zur sexuellen Funktionsfähigkeit, Komorbidität und zu ihrem Hilfesuchverhalten befragt.

Ergebnisse. 9,6\% der Befragten berichten über eine vollständige, 18,0\% über eine moderate und $41,4 \%$ über eine minimale ED. Alle Grade der Störung nehmen mit dem Alter zu und korrelieren mit somatischer bzw. psychischer Komorbidität sowie Medikamenteneinnahme. Nur 3,2\% geben an, schon Mittel zur Verbesserung der Erektionsfähigkeit eingenommen bzw. verwendet zu haben.

Schlussfolgerungen. Nur ein kleiner Teil von Männern mit ED sucht ärztliche Hilfe. Sachliche Aufklärung und kompetente ärztliche Beratung von Betroffenen erscheinen angesichts der guten Wirksamkeit von PDE5-Hemmern angebracht.

Schlüsselwörter

Erektile Dysfunktion · Zweite Lebenshälfte · Komorbidität · Hilfesuchverhalten

\section{Erectile dysfunction in men in their second half of life}

\begin{abstract}
Background. Studies to date on the prevalence of erectile dysfunction (ED) report varying results. The present study investigates the frequency and degree of ED, its determining factors as well as the helpseeking behavior of the patients.

Patients and methods. A random sample of 628 Swiss men (mean age: 61.5 years) were examined by questionnaire regarding their sexual functioning, comorbidity, and help-seeking behavior.

Results. Of the respondents, $9.6 \%$ reported complete, $18.0 \%$ moderate, and $41.4 \%$ minimal ED. All grades of the disorder in-
\end{abstract}

creased with age and correlated with somatic and psychiatric comorbidity as well as with drug intake. Only 3.2\% reported having used drugs for improvement of erectile functioning. Conclusion. Only a small number of men suffering from ED seek the help of a doctor. Considering the efficacy of PDE5 inhibitors, objective information and competent medical counseling of ED patients are indicated.

\section{Keywords}

Erectile dysfunction - Second half of life . Comorbidity $\cdot$ Help seeking behaviour die ,Manneskraft ${ }^{`}$ zurückgehen und die Erektion ist nicht mehr stark genug, um befriedigenden Geschlechtsverkehr zu haben. Unabhängig davon, ob Sie nun Sex mit einer Partnerin haben oder nicht, wie steht es mit der Erektion bei Ihnen? Denken Sie an die letzten 3 Monate und kreuzen Sie bitte diejenige Antwort an, welche am ehesten auf Sie zutrifft." Die Antwortmöglichkeiten waren:

- Ich bin immer fähig, eine Erektion zu bekommen und aufrecht zu erhalten, um Geschlechtsverkehr zu haben.

- Ich bin normalerweise (meistens) fähig, eine Erektion (...).

- Ich bin manchmal fähig, eine Erektion (...).

- Ich bin nie fähig, eine Erektion (...).

\section{Stichprobenrekrutierung}

Um eine möglichst repräsentative Zufallsstichprobe zu erhalten, wurden aus der Telefonadressenkartei der Deutschschweiz nach dem Zufallsprinzip 26.0oo Personen beiderlei Geschlechts gezogen. Diesen wurde ein Brief mit einer Kurzinformation über die Studie zugeschickt sowie eine Ankündigung, dass sie in ca. 1 Woche telefonisch kontaktiert würden. 75 (o,4\%) Personen waren telefonisch nicht erreichbar, in 14.903 Haushalten $(57,3 \%)$ lebte niemand $>45$ Jahre, 6966 Personen $(26,7 \%)$ verweigerten jegliche Auskunft. Mit 4056 $(15,6 \%)$ konnte ein kurzes Telefongespräch geführt werden. Von diesen waren 3418 bereit, den Fragebogen auszufüllen. 1498, davon 641 Männer, schickten einen auswertbaren Fragebogen zurück. 13 beantworteten die Frage nach der Erektionsfähigkeit nicht, sodass eine Stichprobe von 628 Männern im Alter zwischen 45 und 91 Jahren (Mittel $=61,5, \mathrm{SD}=10,3$ Jahre) resultierte. Weitere soziodemographische Merkmale gehen aus $\bullet$ Tabelle 1 hervor.

Die teilnahmebereiten Personen wiesen gegenüber den Verweigerern geringe Unterschiede auf: Sie erachteten Gesundheit und Sexualität als wichtiger für ihre Lebenszufriedenheit, schätzten ihre Gesundheit besser ein, wiesen eine höhere allgemeine Lebenszufriedenheit auf und bezeichneten sich selbst als der Sexualität gegenüber liberaler eingestellt als Gleichaltrige. 


\section{Hier steht eine Anzeige.}

黛 Springer 
Originalien

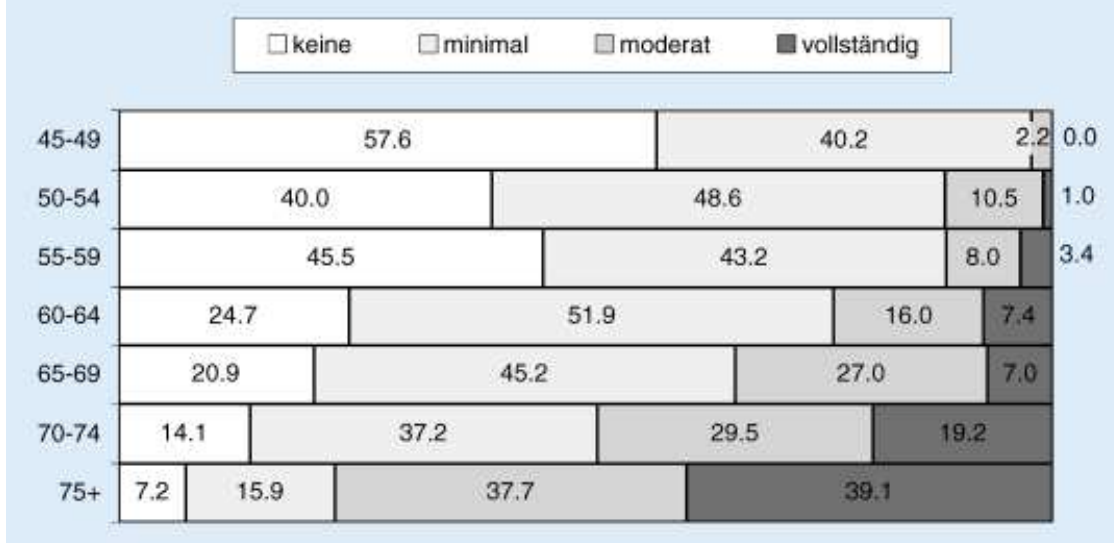

Abb. $1 \Delta$ Grad der ED in den einzelnen Alterskohorten

Die statistischen Auswertungen wurden mit SPSS, Version 11, auf Macintosh durchgeführt. Für die bivariaten Beziehungen wurden Spearman Korrelationen berechnet.

\section{Ergebnisse}

\section{Erektile Dysfunktion und Alter}

Von den 628 Männern geben 195 (31,1\%) an, keinerlei Schwierigkeiten mit der Erektion zu haben. $260(41,4 \%)$ berichten über minimale, 113 (18,o\%) über moderate Erektionsschwierigkeiten und 60 (9,6\%) beklagen einen vollständigen Verlust der ED. Die detaillierten Häufigkeiten für die einzelnen Alterskohorten gehen aus $\bullet$ Abb. 1 hervor.

Es zeigt sich, dass die subjektiv wahrgenommene Einschränkung der Erektionsfähigkeit mit dem Alter kontinuierlich zunimmt. Jenseits des 50. Altersjahres geben über die Hälfte und jenseits des 65. Altersjahres mehr als drei Viertel der Männer eine ED unterschiedlichen Grades an.

\section{Erektile Dysfunktion und Gesundheitsmerkmale}

Zusammenhänge zwischen verschiedenen Gesundheitsvariablen und einer ED sind in - Tabelle 2 zusammengefasst. Die Zusammenhänge wurden im Sinne von gerichteten Hypothesen einseitig überprüft: Je höher die Symptom- bzw. Merkmalsausprägung, desto schwerwiegender die Erektionsstörung.

Die Ergebnisse lassen sich in folgenden Feststellungen zusammenfassen:
- Männer mit schlechter globaler Gesundheitseinschätzung und hohem „body mass index“ (BMI) weisen vermehrt Erektionsstörungen auf.

- Zusammenhänge zwischen ED und körperlichen Beschwerden zeigen sich für Prostatabeschwerden, hohen Blutdruck und Schmerzstörungen.

- Die Einnahme von Medikamenten, welche einerseits auf komorbide Störungen hinweisen, andererseits im Sinne unerwünschter Nebenwirkungen die Erektionsfähigkeit beeinträchtigen, korreliert mit einer Ausnahme mit der Wahrscheinlichkeit einer ED. Mit Antidepressiva wurde kein Zusammenhang gefunden, was darauf zurückzuführen ist, dass lediglich 3,6\% der Befragten angaben, solche Medikamente einzunehmen.

- Sportliche Aktivität (gemessen mit o = nie bis $4=$ mehrmals pro Woche) geht mit weniger Erektionsschwierigkeiten einher.

\section{Erektile Dysfunktion und sexuelles Interesse, sexuelle Aktivität und Zufriedenheit}

Sexualität ist nicht gleichzusetzen mit Erektion und Koitusfähigkeit. Mit zunehmendem Alter gewinnen Zärtlichkeiten, Petting und sexuelle Erlebnisfähigkeit an Bedeutung. Wie aus $\bullet$ Tabelle 3 hervorgeht, korrelieren Erektionsprobleme mit sämtlichen Aspekten des sexuellen Interesses, der sexuellen Aktivität und Zufriedenheit dahingehend, dass sie um so mehr beeinträchtig sind, je ausgeprägter die ED ist.

\section{Erektionsprobleme und Hilfesuchverhalten}

$505(80,4 \%)$ der Teilnehmer gaben an, noch nie daran gedacht zu haben, Hilfsmittel zur Verbesserung der Erektionsfähigkeit einzunehmen bzw. zu verwenden. $102(16,3 \%)$ hatten daran gedacht, aber noch keine konkreten Schritte unternommen und lediglich $20(3,2 \%)$ berichteten, schon Mittel eingenommen bzw. verwendet $\mathrm{zu}$ haben (1 missing case, o,2\%): Sildenafil $(n=10)$, Prostaglandininjektionen $(n=4)$, Hausmittel $(n=2)$ und einmal pornografisches Bildmaterial.

Dichotomisiert man die Antworten zum Hilfesuchverhalten in "gelassene Einstellung" (noch nie daran gedacht, Hilfsmittel zu verwenden, 80,4\%) und „,besorgte Einstellung" (schon daran gedacht oder schon Mittel verwendet, $19,4 \%$ ), so zeigen sich zwischen den "Gelassenen“ und den „Besorgten“ folgende Unterschiede (bivariate Korrelationen, $p>0,01)$ : Besorgte Männer sind älter und haben schwerere Erektionsstörungen. Sie klagen über Langeweile und sind mit ihrer Gesundheit und ihrem Aussehen unzufriedener. Für sie war Sexualität in jüngeren Jahren wichtiger als für „Gelassene“. „Besorgte“ haben weniger sexuelle Lust, haben mehr Versagensängste und berichten, dass ihnen die Ansprüche ihrer Partnerin manchmal zu weit gehen. Sie haben jüngere Partnerinnen als „Gelassene“, dieser Unterschied ist jedoch statistisch nicht signifikant.

\section{Diskussion}

\section{Methodik und Stichprobe}

Bei den befragten Männern handelt es sich im Vergleich zur Durchschnittsbevölkerung um eine Zufallsstichprobe mit relativ guter Gesundheit und offener und liberaler Einstellung zu sexuellen Fragen. Dies bedeutet, dass die Ergebnisse zur sexuellen Funktionsfähigkeit, Aktivität und $\mathrm{Zu}$ friedenheit vermutlich über dem Durchschnitt der Deutschschweizer Männer dieses Alters liegen. Im Vergleich zu den Stichproben der MMAS [20] und der CMS [11] unterscheidet sich unsere Stichprobe (Zurich Study of Sexuality and Aging, ZSSA) in einigen Merkmalen, welche $z$. T. Unterschiede in den Ergebnissen erklären: 
- Durchschnittsalter: ZSSA=61,5 Jahre, MMAS=53,8 Jahre, $\mathrm{CMS}=51,8$ Jahre;

- Partnerstatus - in fester Partnerschaft: $Z$ SSA $=86 \%$, MMAS $=100 \%$, $\mathrm{CMS}=66 \%$;

- Wohnort - Anteil auf dem Land lebender: $Z S S A=49,5 \%$, MMAS und $\mathrm{CMS}=\mathrm{O} \%$.

Für die Erfassung der ED verzichteten wir im Unterschied zur MMAS bewusst auf den Begriff „Impotenz“, da dieser als diskriminierend verstanden werden kann und wählten den Begriff abgestufter „Erektionsfähigkeit“. Wahrscheinlich ist dies, neben dem höheren Durchschnittsalter, eine Erklärung, dass wir mit 51,4\% einen relativ hohen Anteil von Männern mit minimalen Erektionsschwierigkeiten (,ich bin normalerweise - meistens - fähig, eine Erektion zu bekommen und aufrecht zu erhalten ...) haben. Zum Zeitpunkt der Planung unserer Studie lag noch keine validierte deutschsprachige Version des „,international index of erectile function" (IIEF) vor [24] - sie wurde 2003 publiziert [25] - sodass dieses heute anerkannte Instrument noch nicht zur Verfügung stand. In der CMS wurden mit einem Summenscore wesentlich strengere Kriterien für das Vorliegen einer ED gewählt.

\section{Häufigkeit und Ausmaß der erektilen Dysfunktion}

In Übereinstimmung mit den eingangs zitierten Studien $[5,6,7,8,9,10,11,12,13,20$, 21] zeigt sich, dass sowohl die Häufigkeit wie der Schweregrad einer ED mit dem Alter zunimmt. Dieser Befund deutet auf die wichtige Rolle organischer Ursachen bei ED des älter werdenden Mannes hin. Berücksichtigt man die erwähnten Stichprobenunterschiede, so sind die Ergebnisse unserer Studie denen der MMAS [20] ähnlich, insbesondere hinsichtlich einer vollständigen ED (in beiden Studien 9,6\%) und einer moderaten $\mathrm{ED}(\mathrm{ZSSA}=18,0 \%$, MMAS=25,2\%). Lediglich beim Anteil der minimalen ED ist der Anteil in der vorliegenden Studie mit 41,4\% mehr als doppelt so hoch wie in der MMAS (17,2\%). Die 19,2 \% einer ED in der CMS entsprechen vermutlich in etwa den Häufigkeiten der beiden Kategorien „moderat“ und „voll-

Tabelle 1

Alterskohorten und soziodemographische Merkmale der Stichprobe ( $n=628$ Männer)

\begin{tabular}{|c|c|c|}
\hline \multirow[t]{7}{*}{ Alterskohorten } & - 45-49 Jahre & $92(14,6 \%)$ \\
\hline & - 50-54 Jahre & $105(16,7 \%)$ \\
\hline & - 55-59 Jahre & $88(14,0 \%)$ \\
\hline & - 60-64 Jahre & $81(12,9 \%)$ \\
\hline & - 65-69 Jahre & $115(18,3 \%)$ \\
\hline & • 70-74 Jahre & $78(12,4 \%)$ \\
\hline & - 75 Jahre und älter & $69(11,0 \%)$ \\
\hline \multirow[t]{4}{*}{ Wohnort } & - Dorf & $49,5 \%$ \\
\hline & - Kleinstadt & $18,9 \%$ \\
\hline & - Vorort einer Grossstadt & $17,8 \%$ \\
\hline & - Grossstadt & $13,7 \%$ \\
\hline \multirow[t]{4}{*}{ Zivilstand } & - Ledig & $6,6 \%$ \\
\hline & - Verheiratet & $72,0 \%$ \\
\hline & - Geschieden & $7,5 \%$ \\
\hline & - Verwitwet & $13,9 \%$ \\
\hline \multirow[t]{2}{*}{ Partnerstatus $^{\mathrm{a}}$} & - Mit fester Partnerschaft & $86,0 \%$ \\
\hline & - Ohne feste Partnerschaft & $14,0 \%$ \\
\hline Partnerschaftsdauer [Jahre (Range; & - Verheiratete & $1,0-61,0 ; 31,0 ; 13,0$ \\
\hline \multirow[t]{2}{*}{ Mittel; Standardabweichung)] } & $\begin{array}{l}\text { - Nicht eheliche } \\
\text { Partnerschaften }\end{array}$ & $1,0-58,0 ; 8,3 ; 8,4$ \\
\hline & - Insgesamt & $1,0-61,0 ; 27,2 ; 15,0$ \\
\hline \multirow[t]{4}{*}{ Wohnsituation } & - Zusammen mit (Ehe-) & $77,5 \%$ \\
\hline & Partnerin & \\
\hline & - In separater Wohnung & $9,5 \%$ \\
\hline & - Keine Partnerin vorhanden & $14,0 \%$ \\
\hline
\end{tabular}

a Der Partnerstatus zeigt an, ob eine feste Partnerschaft vorhanden ist, die von den Befragten als "Lebenspartnerschaft" bezeichnet wird.

ständig“ der beiden anderen Studien unter Berücksichtigung des niedrigeren Durchschnittsalters in der CMS [21].

Wie die sexualmedizinische Praxis zeigt, sind Männer mit einer partiellen (moderate, minimale) ED besonders sensibel für zusätzliche psychosoziale Störfaktoren wie z. B. Qualität der Partnerbeziehung, Altersdifferenz zur Partnerin und deren sexuelle Erwartungen. Die Unterscheidung zwischen „rein“ organisch bzw. psychogen bedingten Erektionsstörungen ist gerade bei Männern in der zweiten Lebenshälfte mehr oder weniger theoretisch. Tatsache ist, dass eine organisch bedingte Verschlechterung der Erektionsphysiologie von den Betroffenen subjektiv unterschiedlich wahrgenommen und bewertet werden kann. Dasselbe gilt auch für ihre Partnerinnen. Deshalb sollte bei der Sexualanamnese nicht nur nach „harten Fakten“ wie Frequenz des Geschlechtsverkehrs und Rigidität des Penis, sondern auch nach „weichen Aspekten“ wie der Wahrnehmung und subjektiven Bewertung einer verminderten Erektionsfähigkeit sowie damit zusammenhängenden Ängsten gefragt werden [19].

\section{Somatische und psychische Komorbidität}

Die Ergebnisse entsprechen denen anderer Studien, dass v. a. Erkrankungen des Urogenitaltraktes, insbesondere der Prostata, das Vorliegen einer arteriellen Hypertonie sowie Schmerzstörungen des muskuloskelettalen Systems die Prävalenz einer ED erhöhen $[4,5,6,9,10,11]$. Dies weist auf die mit dem Alter zunehmende Bedeutung somatischer Faktoren in der Ätiologie einer ED hin. Interessant ist der Befund, dass die Korrelationskoeffizienten zwischen den einzelnen Medikamentengruppen und einer ED höher sind als mit den Beschwerdekategorien (s. - Tabelle 2). Dies 
Tabelle 2

Zusammenhänge zwischen ED und Gesundheitsmerkmalen

(Spearman-Korrelationen für ordinale Daten)

\begin{tabular}{llrl} 
& \multicolumn{1}{l}{$\boldsymbol{n}$} & \multicolumn{1}{l}{$\boldsymbol{r}$} & $\boldsymbol{p}_{\text {einseitig }}$ \\
Subjektive Gesundheitseinschätzung & 607 & $-0,25$ & 0,000 \\
\hline BMI & 624 & 0,13 & 0,000 \\
Beschwerden & & & \\
- Rücken- und Kreuzschmerzen & 623 & $-0,06$ & 0,087 \\
- Allgemeine Schwäche & 623 & 0,01 & 0,485 \\
- Bauch/Magenprobleme & 623 & $-0,02$ & 0,312 \\
- Verdauungsprobleme & 623 & 0,04 & 0,151 \\
- Schlafstörungen & 623 & 0,02 & 0,333 \\
- Kopfschmerzen & 623 & $-0,11$ & 0,003 \\
- Herzprobleme & 623 & 0,04 & 0,153 \\
- Brustschmerzen & 623 & 0,06 & 0,070 \\
- Fieber & 623 & $-0,05$ & 0,094 \\
- Gelenk- und Gliederschmerzen & 623 & 0,12 & 0,002 \\
- Nervosität oder Unruhe & 623 & $-0,09$ & 0,014 \\
- Hoher Blutdruck & 623 & 0,16 & 0,000 \\
- Niedriger Blutdruck & 623 & $-0,01$ & 0,444 \\
- Harnlösen, Prostatabeschwerden & 623 & 0,17 & 0,000 \\
\hline Medikamentenkonsum & & & \\
- Schmerzmittel & 616 & 0,07 & 0,044 \\
- Herz-Kreislauf-Medikamente & 616 & 0,22 & 0,000 \\
- Antidepressiva & 616 & 0,04 & 0,154 \\
- Beruhigungsmittel & 616 & 0,12 & 0,002 \\
- Bluthochdruckmittel & 616 & 0,22 & 0,000 \\
- Schlafmittel & 616 & 0,15 & 0,000 \\
- Summenindex Medikamentenkonsum & 616 & 0,29 & 0,000 \\
- Sportliche Aktivität & 621 & $-0,23$ & 0,000 \\
- Alter & 628 & 0,50 & 0,000 \\
\hline
\end{tabular}

Tabelle 3

Zusammenhänge zwischen ED und Aspekten des sexuellen Interesses, Aktivität und Zufriedenheit (Spearman-Korrelationen für ordinale Daten)

\section{Sexuelles Interesse \\ - Wunsch nach Zärtlichkeiten \\ - Wunsch nach Petting \\ - Wunsch nach Geschlechtsverkehr \\ - Sexuelles Verlangen \\ - Gedanken, Phantasien, Träume}

Sexuelle Aktivität

- Zärtlichkeiten

- Petting

- Geschlechtsverkehr

- Selbstbefriedigung

Sexuelle Zufriedenheit

- Genuss Zärtlichkeiten

- Genuss Petting

- Genuss Geschlechtsverkehr

- Allgemeine Zufriedenheit mit Sexualleben

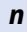

$\mathbf{r}$

$\begin{array}{lll}606 & -0,23 & 0,000 \\ 592 & -0,23 & 0,000 \\ 599 & -0,46 & 0,000 \\ 616 & -0,47 & 0,000 \\ 615 & -0,42 & 0,000\end{array}$

616

611

614

607

548

439

467

604
$-0,19$

$-0,22$

$-0,49$

$-0,24$

0,000

0,000

0,000

0,000

$\begin{array}{ll}-0,16 & 0,000 \\ -0,10 & 0,023 \\ -0,27 & 0,000 \\ -0,32 & 0,000\end{array}$

lässt sich damit erklären, dass wahrscheinlich nur diejenigen Personen, welche ausgeprägtere Beschwerden hatten, medikamentös behandelt wurden. Klinisch von Bedeutung ist, dass sich die sexualphysiologisch hemmende Wirkung von Komorbiditäten und Medikamentennebenwirkungen oft addieren.

\section{Sexuelles Interesse und sexuelle Zufriedenheit}

Für die Lebensqualität vieler älterer Menschen hat die sexuelle Funktionsfähigkeit und Zufriedenheit einen hohen Stellenwert, wie auch unsere Ergebnisse zeigen. Dies gilt auch für älter werdende Frauen, wie die Resultate des dieser Arbeit zugrunde liegenden umfangreichen Forschungsprojekts belegen [23]. Daraus folgt, dass ältere Frauen heute nicht mehr passiv auf sexuelle Initiativen ihrer Partner warten, sondern selbst sexuelle Wünsche offen äußern. Vor allem Männer, die eine partielle ED besorgt wahrnehmen und erleben, laufen Gefahr, in ihrem Selbstwertgefühl verunsichert zu werden. In unserer Stichprobe war jeder 5. Mann über seine Erektion besorgt. Diese Besorgnis verbirgt sich oft hinter anderen, als gravierend geklagten Körperbeschwerden.

\section{Hilfesuchverhalten}

Lediglich 20 Männer (3,3\% !!) berichteten, dass sie schon Mittel eingenommen bzw. verwendet hätten zur Verbesserung ihrer ED. Diese Zahl liegt deutlich unter der in der multinationalen MALES-Studie ermittelten Zahl von 16\% der Männer mit einer ED, welche zum Zeitpunkt der Befragung einen PDE-5-Hemmer einnahmen [17]. Mögliche Erklärungen für diese Differenz sind, dass die Daten unserer Studie 1999 und damit früher als die der MALES-Studie erhoben wurden und dass vermutlich der Anteil der ländlichen Bevölkerung mit eher traditioneller Sexualeinstellung höher ist.

Ein anderer Aspekt sollte bei der Erklärung des zurückhaltenden Hilfesuchverhaltens von Männern mit ED ebenfalls berücksichtigt werden. Die etwa gleich bleibende sexuelle Zufriedenheit älter werdender Frauen und Männer [23], trotz abnehmender sexueller Funktionsfähigkeit, lässt 
sich mit dem aus der Sozialpsychologie bekannten Phänomen des sog. „response shift “ [26] teilweise erklären. Damit ist gemeint, dass man mit abnehmender Vitalität und körperlicher Gesundheit in der Sexualität mit weniger zufrieden ist. Oder anders formuliert: Die genitale Dimension der Sexualität scheint mit zunehmendem Alter bei beiden Geschlechtern an Bedeutung zu verlieren zugunsten einer Sexualität, die sich in Zuneigung, körperlichen Zärtlichkeiten und spielerischem Petting äußern kann [23].

\section{Fazit für die Praxis}

Ungefähr jeder 5. Mann in der zweiten Lebenshälfte hat eine mittelgradige bis schwere ED. Nur ein kleiner Teil dieser Männer nimmt dies zum Anlass, einen Arzt aufzusuchen. Eine partielle, und damit häufig situativ unter zusätzlichen psychischen Belastungen auftretende ED ist etwa 4-mal häufiger als ein vollständiger Erektionsverlust. Der geringe Anteil von Männern mit einer ED, welche schon Behandlungserfahrung mit einem PDE-5Hemmer haben, weist darauf hin, dass kompetente Aufklärung und Beratung über die heutigen Behandlungsmöglichkeiten einer ED nicht nur in der ärztlichen Praxis, sondern auch in der breiten Bevölkerung dringend angezeigt sind.

\section{Korrespondierender Autor Prof. Dr. C. Buddeberg}

Abteilung Psychosoziale Medizin, Universitätsspital,

Haldenbachstraße 18, CH-8091 Zürich, Schweiz E-Mail: claus.buddeberg@usz.ch

Interessenkonflikt: Der korrespondierende Autor versichert, dass keine Verbindungen mit einer Firma, deren Produkt in dem Artikel genannt ist, oder einer Firma, die ein Konkurrenzprodukt vertreibt, bestehen.

\section{Literatur}

1. Masters WH, Johnson VE (1966) Human sexual response. Little \& Brown, Boston

2. Masters WH, Johnson VE (1970) Die sexuelle Reaktion. rororo Sexologie, Reinbeck

3. Prins J, Blanker MH, Bohnen AM et al. (2002) Prevalence of erectile dysfunction: a systematic review of population-based studies. Int J Impot Res 14: 422-432
4. Kubin M, Wagner G, Fugl-Meyer AR (2003) Epidemiology of erectile dysfunction. Int J Impot Res 15: 63-71

5. Rosen R, Fisher W, Eardley J et al. (2004) The multinational men's attitudes to life events and sexuality (MALES) study: I. Prevalence of erectile dysfunction and related health concerns in the general population. Curr Med Res Opin 20: 607-617

6. Lewis RW, Fugl-Meyer KS, Bosch R et al. (2004) Epidemiology/Risk Factors of Sexual Dysfunction. J Sex Med 1: 35-39

7. Aytac IA, Araujo AB, Johannes CB et al. (2000) Socioeconomic factors and incidence of erectile dysfunction: Findings of the longitudinal Massachusetts Male Aging Study. Soc Sci Med 51: 771-778

8. Tengs T, Osgood ND (2001) The link between smoking and impotence: Two decades of evidence. Prev Med 32: 447-452

9. Braun MH, Sommer F, Haupt G et al. (2003) Lower urinary tract symptoms and erectile dysfunction: co-morbidity or typical „Aging Male" symptoms? Results of the "Cologne Male Survey". Eur Urol 44: 588-594

10. Rosen RC, Altwein J, Boyle P et al. (2003) Lower urinary tract symptoms and male sexual dysfunction: the multinational survey of aging male (MSAM-7). Eur Urol 44: 637-649

11. Braun M, Sommer F, Lehmacher W et al. (2004) Erectile dysfunction. Are interdisciplinary diagnosis and therapy necessary? Dtsch Med Wochenschr 129: $131-136$

12. Araujo AB, Durante R, Feldman HA et al. (1998) The relationship between depressive symptoms and male erectile dysfunction: Cross-sectional results from the Massachusetts Male Aging Study. Psychosom Med 60: 458-465

13. Okulate G, Olayinka O, Dogunro AS (2003) Erectile dysfunction: Prevalence and relationship to depression, alcohol abuse and panic disorder. Gen Hosp Psychiatry 25: 209-213

14. Ventegodt $S$ (1998) Sex and the quality of life in Denmark. Arch Sex Behav 27: 295-307

15. Beutel ME, Schumacher J, Weidner W et al. (2002) Sexual activity, sexual and partnership satisfaction in aging men-results from a German representative community study. Andrologia 34: 22-28

16. Moore TM, Strauss JL, Herman S et al. (2003) Erectile dysfunction in early, middle, and late adulthood: Symptom patterns and psychosocial correlates. J Sex Marital Ther 29: 381-399

17. Fisher WA, Rosen RC, Eardley J et al. (2004) The Multinational Men's Attitudes to Life Events and Sexuality (MALES) Study, Phase II: Understanding PDE-5 inhibitor treatment seeking patterns, among men with erectile dysfunction. J Sex Med 1: 150-160

18. Nehra A, Kulaksizoglu H (2002) Global perspectives and controversies in the epidemiology of male erectile dysfunction. Curr Opin Urol 12: 493-496

19. Buddeberg C (2005) Sexualberatung. Eine Einführung für Ärzte, Psychotherapeuten und Familienberater, 4. überarb. Aufl. Thieme, Stuttgart

20. Feldman HA, Goldstein I, Hatzichristou DG et al. (1994) Impotence and its medical and psychosocial correlates: Results of the Massachusetts Male Aging Study. Urology 151: 54-61

21. Braun M, Wassmer G, Klotz T et al. (2000) Epidemiology of erectile dysfunction: results of the "Cologne Male Survey". Int J Impot Res 12: 305-311

22. Bucher T, Hornung R, Gutzwiller F et al. (2001) Sexualität in der zweiten Lebenshälfte. Erste Ergebnis se einer Studie in der deutschsprachigen Schweiz. In: Beberich H, Brähler E (Hrsg) Sexualität und Partnerschaft in der zweiten Lebenshälfte. Psychosozial-Verlag, Gießen, S 31-59
23. Bucher T, Hornung R, Buddeberg C (2003) Sexuelle Zufriedenheit in der zweiten Lebenshälfte. Ergebnisse einer empirischen Untersuchung. Z Sexualforschung 16: 249-270

24. Rosen RC, Riley A, Wagner G et al. (1997) A multidimensional scale for assessment of erectile dysfunction. Urology 49: 822-830

25. Wiltink J, Hauck W, Phädayanon M et al. (2003) Validation of the German version of the International Index of Erectile Function (IIEF) in patients with erectile dysfunction, Peyronie's disease and controls. Int J Impot Res 15: 192-197

26. Sprangers MA, Schwartz CE (1999) Integrating response shift into health-related quality of life research: a theoretical approach. Soc Sci Med 48: 15071515 